\title{
Deformations and Thermal Stability of Carbon Nanotube Ropes
}

\author{
María J. López $^{a}$, Angel Rubio ${ }^{b}$, and Julio A. Alonso ${ }^{c}$ \\ ${ }^{a}$ Departamento de Física Teórica, Universidad de Valladolid, 47011 Valladolid, Spain \\ ${ }^{b}$ Departamento de Física de Materiales, Universidad del País Vasco and Donostia \\ International Physics Center, DIPC, 20018 San Sebastián/Donostia, Spain \\ ${ }^{c}$ Departamento Física Teórica, Universidad de Valladolid, 47011 Valladolid, Spain and \\ Donostia International Physics Center, DIPC, 20018 San Sebastián/Donostia, Spain
}

\begin{abstract}
Structural and thermal characteristics of crystalline ropes of single-wall carbon nanotubes (SWCNTs) are investigated. Novel crystalline ropes of polygonized SWCNTs produced by laser irradiation exhibit rounded-hexagonal cross sections in contrast to earlier observations of circular tubes. Extensive molecular dynamics (MD) simulations lead to several metastable structures of the lattice characterized by different tube cross sections, hexagonal, rounded-hexagonal and circular, and increasing cell volume. The competition between different tube shapes is analyzed and compared to experiments. On the other hand, bundles of SWCNTs coalesce forming multiwall carbon nanotubes (MWCNTs) under thermal treatment at high temperatures. Extensive MD simulations confirm the SW-to-MW transformation and suggest the physical patching-and-tearing mechanism underlying the concerted coalescence of the tubes.
\end{abstract}

Keywords: carbon nanotubes, ropes, deformations, coalescence

\section{INTRODUCTION}

As it is well known, carbon nanotubes (CNT) are seamless cylinders of $s p^{2}$ carbon atoms arranged in a graphitic honeycomb structure. Carbon nanotubes may be one fold $\left(\mathrm{SWCNTs}^{1}\right)$ or contain several cylinders nested one inside another $\left(\mathrm{MWCNTs}^{2}\right.$ ). SWCNTs, in many cases, self-organize into crystalline bundles ${ }^{3,4}$ (set of a few to a few hundred aligned tubes arranged in a two-dimensional triangular lattice in the plane perpendicular to their common axes). Although these metastable forms of carbon are subjected to intensive investigations there are still important gaps in our understanding and control of the properties of these materials. A thorough understanding of carbon nanotubes includes the detailed and comprehensive characterization of their possible deformations due to interactions with a substrate or with other tubes, and of their stability under thermal treatment or chemical agents.

It has been shown that CNTs may deform elastically away from their ideal circular cross sections when they interact either with a substrate ${ }^{5}$ or with other tubes. ${ }^{6,7}$ Deformations of the tubes may have non trivial effects in their properties such as conductivity or phonon spectrum. Tubes in a bundle interact one to another through attractive van der Waals forces, similar to the ones acting between graphene layers in graphite. Therefore, the intertube interaction could prompt elastic structural changes in the tubes. We have being able to synthesize novel crystalline bundles of "polygonized" (with hexagonal cross sections) SWCNT's. ${ }^{8}$ Our finding opens up the question of what is the equilibrium configuration of a lattice of aligned tubes and the possibility of the existence of several metastable structures which could be obtained depending on the growth conditions. To shed some light on this problem we have performed extensive molecular dynamics simulations of lattices of monodisperse armchair and zigzag SWCNTs as a function of tube diameter. We find several metastable structures of the lattice characterized by different tube cross sections, hexagonal, rounded-hexagonal and circular, and increasing cell volume. Our results presented in Section 3 help to interpret both, the earlier (circular) and the presently (hexagonal) observed tube shapes.

Send correspondence to M.J.L.; E-mail: maria@lab2.fam.cie.uva.es 
On the other hand, since CNTs are metastable (the most stable form of carbon is graphite), they may transform into more stable structures under the appropriate annealing conditions. We have found that bundles of SWCNTs coalesce forming MWCNTs, containing from two to six nested tubes, under thermal treatment at high temperatures. ${ }^{9-11}$ This structural transformation is confirmed by extensive Molecular Dynamics (MD) ${ }^{11}$ simulations presented in Section 4. The simulations combined with the experiments suggest a "patching-andtearing" mechanism for the SW to MWCNT's transformation underlying the "concerted" coalescence of the tubes that begins with their polymerization.

\section{THEORETICAL MODEL}

Due to the large number of atoms (up to 6000 nonequivalent atoms) and the large time scales (of the order of 1000 ps) involved in the simulation of the structural and thermal properties of SWCNT bundles, the use of accurate, ab initio quantum techniques in the description of these systems becomes impractical if not completely unfeasible. Therefore, we use a reliable and computational efficient, many-body interatomic potential to mimic the carboncarbon interactions. The potential consist in two terms: i) a short-range part which is well suited for describing the intratube covalent bonds ${ }^{12,13}$ and ii) a long range term which describes the van de Waals interactions between adjacent tubes. ${ }^{14}$ This potential appropriately describes both, the covalent bond in diamond and graphite and the van der Waals attraction between graphene layers in graphite. It has been successfully applied to the study of fullerenes and tubes.

The dynamical simulations are performed within the constant-energy constant-volume ensemble. The time evolution of the system is obtained by numerical integration, using the velocity version of the Verlet algorithm, of the classical Newtonian equations of motion. To investigate possible metastable structures of the lattice of SWCNTs a number of high energy configurations (for several lattice parameters and various tube shapes) are generated and relaxed using the thermal quenching procedure. This procedure consists in the step-wise removal of the kinetic energy of the system, along a dynamical trajectory, until the forces on all the atoms vanish and a local minimum on the potential energy surface has been found. The lowest energy minimum found will, likely, correspond to the equilibrium configuration of the lattice and several metastable structures will be also obtained. We have also investigated the thermal behavior of bundles of SWCNTs heat treated at high temperatures. The simulation begins with cool tubes which are progressively heated up by scaling up the velocities of all the atoms.

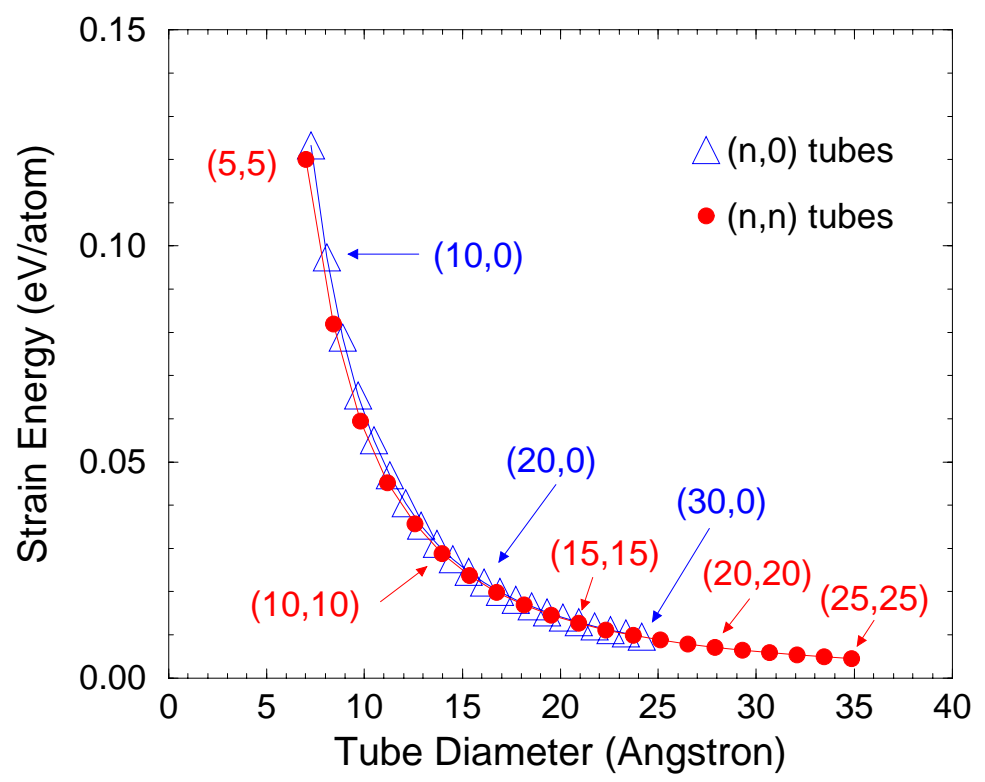

Figure 1. Strain energy of armchair $(n, n)$ and zig-zag $(n, 0)$ SWCNTs as a function of tube diameter. 
The temperature of the bundles is raised up to a value $\left(3000-3500^{\circ} \mathrm{C}\right)$ such that the rate of coalescence is high enough for being observed within the time scale $(t \leq 1000 \mathrm{ps})$ of our simulations.

\section{POLYGONIZED ROPES}

Novel crystalline ropes of polygonized SWCNTs have been produced by $\mathrm{CO}_{2}$ laser ablation. ${ }^{8}$ The ropes consist of monodisperse SWCNTs with tube diameters $D$ of approximately $17 \AA$ arranged in a triangular lattice. HRTEM images of the lattice ${ }^{8}$ clearly show the departure of the tubes from the circular cross section and the appearance of facets, parallel one to another, between adjacent tubes. The polygonized, hexagonal, cross section of the tubes is compatible with the two-dimensional triangular symmetry of the lattice. Our finding of "hexagonal" tubes is in contrast to previous observations of tubes with almost circular cross sections. ${ }^{15}$ Then the question arises about the lowest energy structure of the lattice of aligned tubes and the possibility of having several metastable stuctures depending on the growth conditions. To gain some insight into these problems we have performed an extensive search of possible metastable structures of the (triangular) lattice of aligned tubes. Eventually, the equilibrium configuration of the lattice will be also found.

It is well established that free standing isolated tubes present circular cross sections, since the circular shape minimizes the strain energy of the tubes. (In this context the strain energy is the elastic energy needed to roll up a graphene sheet into a cylinder to form a nanotube.) Figure 1 shows the strain energy of armchair and zigzag SWCNTs as a function of tube diameter $D$. This energy decreases as $1 / R^{2}(R$ is the tube radius) as expected from the continium elastic model. ${ }^{16}$ The tubes, however, may deform elastically when they interact either with a substrate or with other tubes. Therefore, tubes in a bundle may depart, due to the intertube van der Waals-type interaction, from the circular shape. The deformation energy, i.e. energy cost of changing the cross section of the tubes from the ideal circular shape, should be, then, compensated by the intertube interaction energy which acts as the driving force for the elastic structural changes of the tubes. Figure 2 shows the deformation (polygonization) energy of armchair hexagonal tubes, the intertube interaction energy between those tubes when forming a triangular lattice, and the cohesive energy of that lattice of hexagonal tubes with

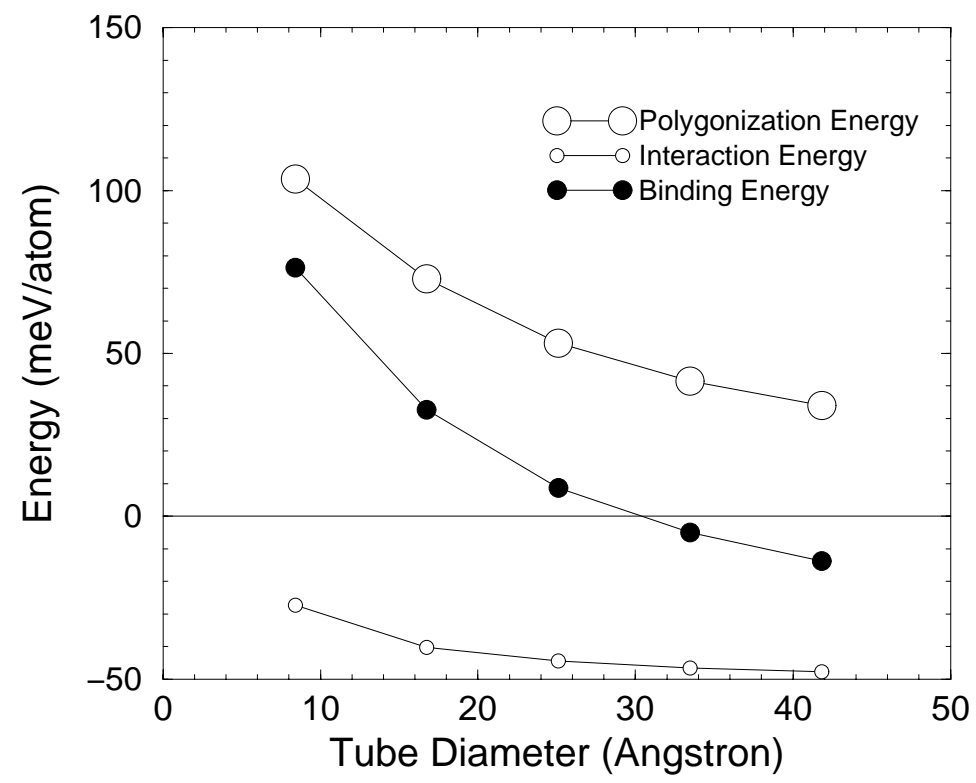

Figure 2. Cohesive energy, with respect to the isolated circular tubes, and intertube interaction energy of a lattice of hexagonal $(n, n)$ tubes as a function of tube diameter. Also shown the polygonization energy of the (n,n) hexagonal tubes. (See text for details). 


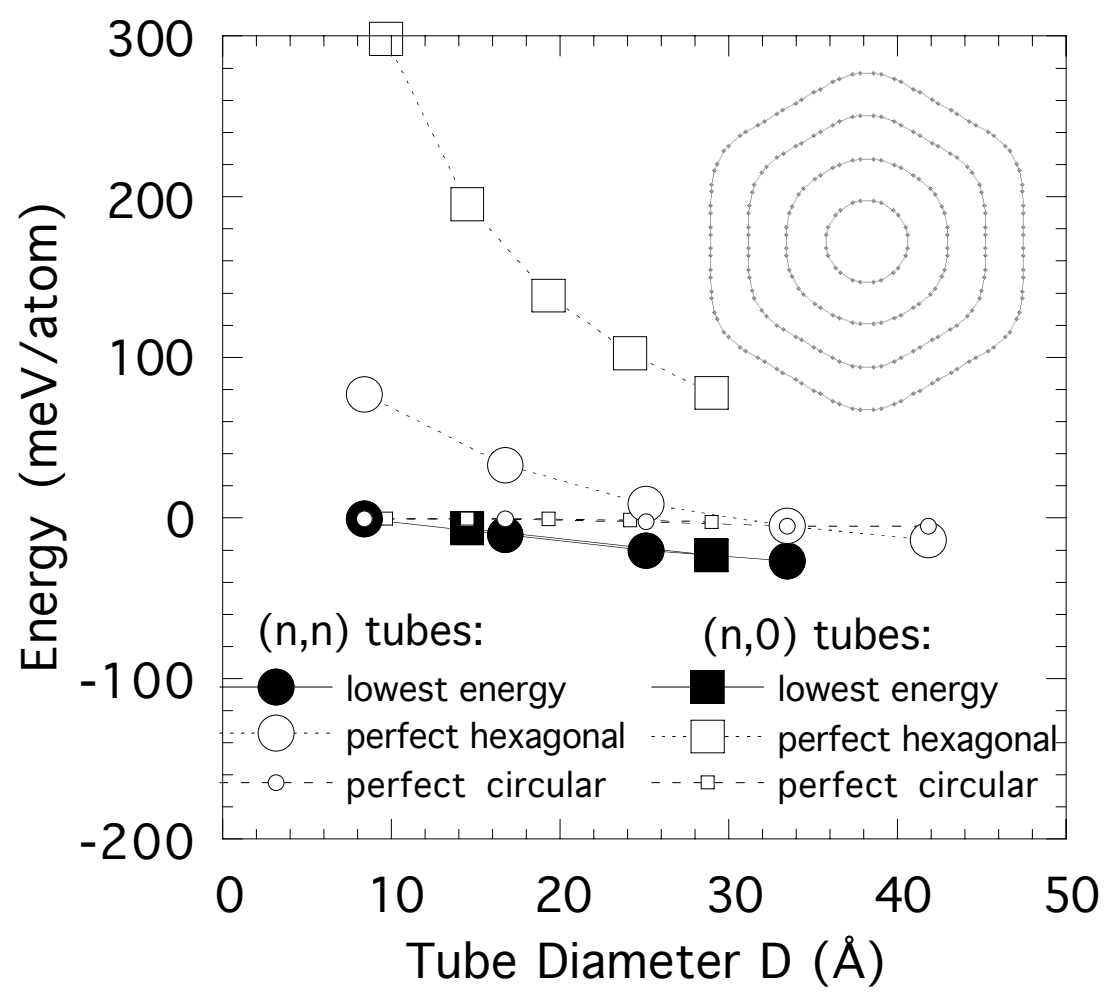

Figure 3. Cohesive energy, with respect to the isolated circular tubes, of the triangular lattice of i) circular, ii) hexagonal, and iii) lowest energy tubes as a function of tube diameter. (n,n) armchair and $(n, 0)$ zigzag tubes are considered. The inset shows, superimposed, the structures of the lowest energy $(n, n)$ tubes.

respect to the isolated circular tubes, all as functions of tube diameter $D$. The balance between the deformation energy, which opposes the polygonization of the tubes in the lattice, and the intertube interaction energy yields to stable lattices of hexagonal armchair tubes for tube diameters larger than $30 \AA$.

The lattice of circular tubes is always a bound system irrespective of the diameter of the constituent tubes (see Fig. 3). Its cohesive energy, however, is pretty small as compared to the interaction energy between graphene layers in graphite. The reason being in the lack of planar interacting faces between adjacent tubes. Notice that the interaction energy between hexagonal tubes, which exhibit planar facets lying parallel one to another between adjacent tubes, is 1 order of magnitude larger than that of the lattice of circular tubes (see Fig. 2). However, for small diameter tubes, this energy does not compensate the high deformation energy of hexagonal tubes and the lattice of circular tubes is more stable than the corresponding lattice of hexagonal tubes. Eventually, since the deformation energy decreases with increasing tube diameter, the lattice of hexagonal tubes becomes more stable than the lattice of circular tubes (see Fig. 3). The onset of polygonization, $D=34$ and $50 \AA$ for armchair and zigzag tubes, respectively, is, however, too high as compared with the experimental value, $D=17 \AA$.

To obtain metastable, and eventually the lowest energy, structures of the lattice of tubes we have applied the thermal quenching procedure. First, a number of lattice configurations corresponding to several values of the lattice parameter and to tube shapes ranging from circular to hexagonal are generated. Then, all those high energy configurations of the lattice are cool down into rigid structures corresponding to local minima of the potential energy surface. The relaxations yield several metastable structures characterized by different tube cross sections and unit cell volume. The shape of the tubes changes, with increasing cell volume, from "nearly" hexagonal to "almost" circular going through an intermediate volume lattice formed by hexagonal tubes with rounded corners. The lowest energy configuration of the lattice evolves from circular to hexagonal tubes with increasing tube diameter. For $D \leq 17 \AA$ nearly circular tubes are found. This explains why all previous 


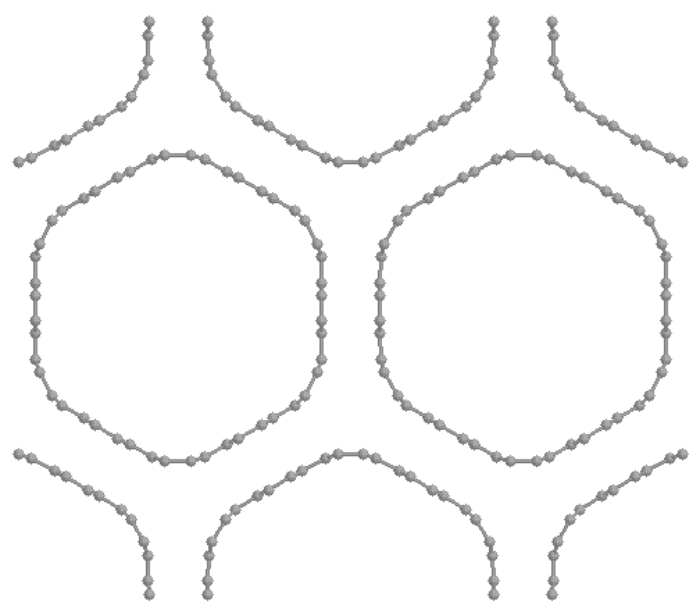

Figure 4. Metastable structure of a lattice of $(12,12)$ tubes with rounded hexagonal cross section. This structure supports the novel crystalline ropes of polygonized tubes produced by $\mathrm{CO}_{2}$ laser ablation.

observations of bundles of tubes in this size range show tubes with circular cross sections.

However, the metastable structure, shown in Figure 4, formed by rounded hexagonal tubes is only 0.6 $\mathrm{meV} /$ atom above the circular lowest energy structure of $(12,12)$ tubes. Similarly the metastable structure of rounded hexagonal $(18,0)$ tubes is only $2.8 \mathrm{meV} /$ atom above the minimum. These novel metastable structures give support to the crystalline bundles of polygonized tubes found in the experiment.

\section{THERMAL TRANSFORMATIONS OF SWCNT ROPES}

Coalescence of two SWCNTs into a larger diameter SWCNT has been observed upon the annealing of SWCNTs at high temperatures either in the presence of $\mathrm{H}_{2}{ }^{17}$ or under electron irradiation. ${ }^{18}$ We have discovered, ${ }^{11}$ as we were investigating the stability of SWCNT ropes under thermal treatment, a new structural transformation of bundles of SWCNTs into MWCNTs. Samples * of SWCNT ropes produced by the catalytic arc-discharge technique ${ }^{4}$ were heated for about $15 \mathrm{~min}$. at temperatures between $1600-2800^{\circ} \mathrm{C}$ under argon flow. Above $2200^{\circ} \mathrm{C}$ the bundles disappear giving rise to MWCNTs consisting of two to six nested tubes. All the characterization techniques of the samples, i.e., High Resolution Transmission Electron Microscopy (HRTEM), X-ray diffraction and Raman spectroscopy, confirm the structural reorganization of the bundles. This SW-to-MW transformation is also confirmed by extensive Molecular Dynamics (MD) simulations (see below). The simulations combined with the experimental results unveil the physical "patching-and-tearing" mechanism of this showy transformation. This novel mechanism is of general applicability and describes both the coalescence of two SWCNTs into a larger diameter SWCNT and the newest transformation of SWCNT ropes into MWCNTs.

\subsection{Coalescence of two tubes}

First we study the temperature induced coalescence of two tubes. The presence of defects in the tubes may act as a driving force for coalescence. It has been shown that vacancies are the leading defects promoting coalescence. ${ }^{18}$ Vacancies can be produced by chemical treatment, ${ }^{17}$ electron irradiation, ${ }^{18}$ etc. Certainly, the thermal treatment of the tubes at relatively high temperatures (above $1600^{\circ} \mathrm{C}$ ) will produce the required vacancies in the tubes. Due to the high temperatures, the vacancies have a high mobility and diffuse throughout the tube until they get locked in the intertube region by saturation of the associated dangling bonds with the dangling bonds left free by a nearby vacancy in the adjacent tube. Saturation of dangling bonds between neighboring tubes yields to intertube polymerization which is the initial stage for the coalescence of tubes. At

${ }^{*}$ HRTEM characterization of the samples shows that they are made of bundles of SWCNTs with bundle diameters ranging from 5 to $20 \mathrm{~nm}$. The individual tubes of about $1.4 \mathrm{~nm}$ in diameter are arranged in a triangular lattice. 

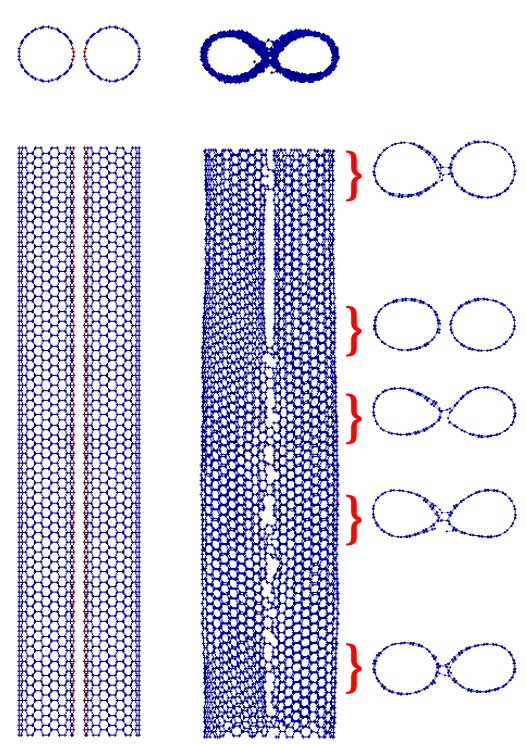

$\mathrm{t}=0 \mathrm{ps}$

$\mathrm{t}=2 \mathrm{ps}$
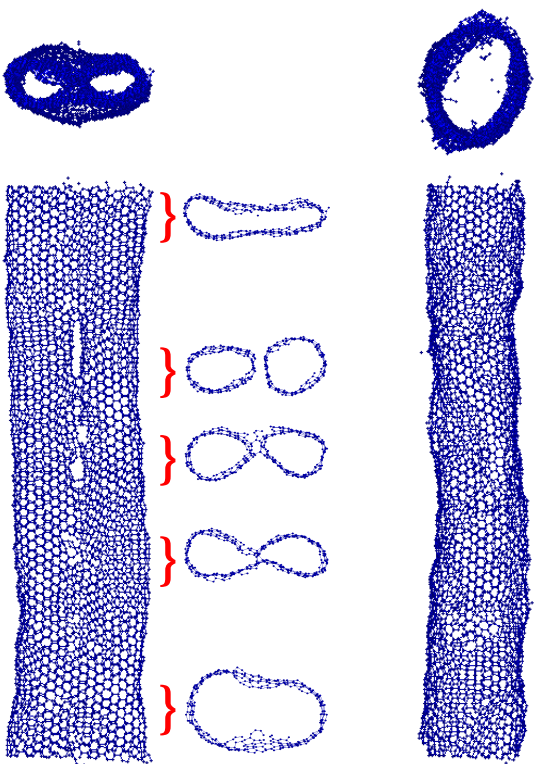

$\mathrm{t}=125 \mathrm{ps}$

$\mathrm{t}=165 \mathrm{ps}$

Figure 5. Snapshots (side and top views) showing the sequence of the coalescence of two $(10,10)$ tubes. Also shown top views of some tube portions embraced by brackets.

the experimental temperatures, the creation of vacancies in the tubes requires heat treatments of about minutes. This time scale is clearly not tractable in conventional MD simulations. However, once a sufficient number of intertube links are formed, coalescence proceeds within a time scale of a few hundred picoseconds. It is this second part of the process which is suitable for being simulated in the computer and therefore we concentrate our study in this part.

We have simulated the coalescence of two $(10,10)$ tubes under thermal treatment (see Fig. 5). The initial intertube distance between the tubes is of $17 \AA$. The first step consists in the generation of vacancies by random removal of a number $(2 \%)$ of atoms. Since no significant diffusion of vacancies is expected within the time scale of the simulations, only those vacancies in the vicinity of the partner tube play an active role in the coalescence process. Therefore we restrict the removal of atoms to the intertube region. Moreover, long simulation cells, containing 60 unit cells in the axial direction of the tubes, are considered to avoid unphysical uniform distributions of vacancies arising from the use of periodic boundary conditions in the axial direction of the tubes. Polymerization of the two tubes occurs very rapidly (in $2 \mathrm{ps}$ ) after the generation of vacancies. The formation of intertube links is driven by the saturation of the dangling bonds left free by the vacancies which results in a non uniform distribution of the links along the tubes length (see Fig. 5, $t=2 \mathrm{ps).} \mathrm{By} \mathrm{progressively} \mathrm{heating} \mathrm{up}$ the tubes, the initial intertube links develop into enveloping surfaces, or "patches", common to the two tubes. The "tearing" apart of the intratube bonds in the intertube region in some of the patches gives rise to partial coalescence of the tubes whereas other portions of the tubes remain polymerized or even completely unlinked (see Fig. $5, t=125 \mathrm{ps}$ ). As the time evolves, new intertube links and patches develop, the existing patches and the coalesced parts of the tubes grow in the axial direction and merge one to another, till, eventually, all the remaining intratube bonds in the intertube region "tear" apart producing the coalesce of the two tubes in their whole length (see Fig. 5, $t=165 \mathrm{ps}$ ). The patching-and-tearing mechanism emerging from our simulations, explains the coalescence of two tubes in great detail. This mechanism gives support to the early intuitive "zipping" description of coalescence. ${ }^{17}$ Moreover, our results on the coalescence of two $(10,10)$ tubes, are in fair agreement with the tight-binding simulations of Terrones et al.. ${ }^{18}$

We have also found, in contrast to the earlier statement ${ }^{18}$ that this process was very unlikely, that coalescence 

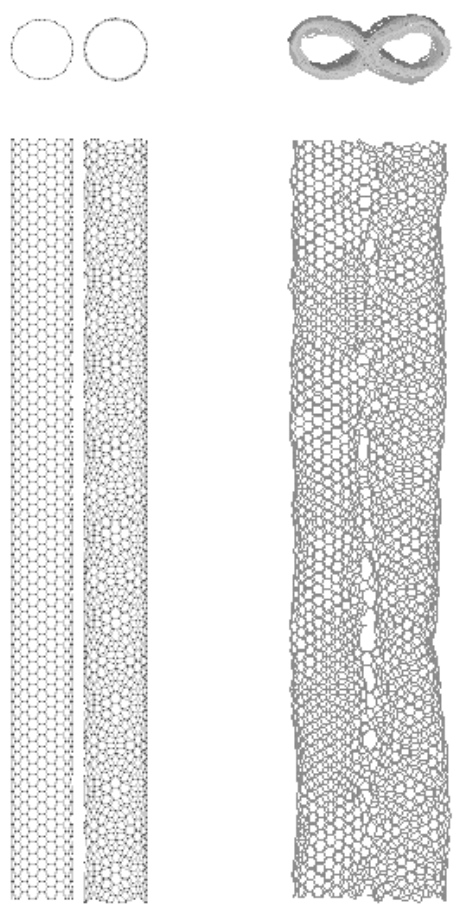
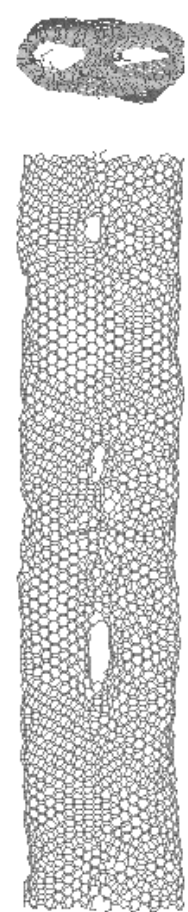
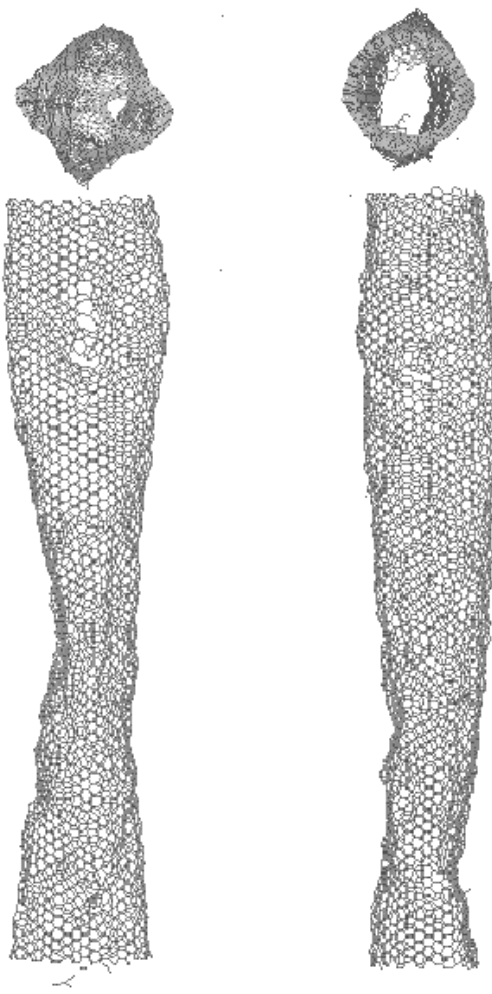

Figure 6. Snapshots (side and top views) showing the sequence of the coalescence of a $(10,10)$ and a $(12,8)$ tubes.

proceeds between tubes of different chiralities without any restriction. Figure 6 shows the coalescence of a $(10,10)$ and a $(12,8)$ tubes. The two tubes have similar radii and a long simulation cell containing approximately (due to the incommensurability of the two tubes) 68 and 9 unit cells, respectively, has been used in the simulations. We find coalescence of the tubes even in the most stringent case of two non chiral tubes one of the armchair type, the $(10,10)$ tube, and the other one of the zigzag type, the $(17,0)$ tube. For both pairs of tubes, large tube reconstructions are required for knitting the two corresponding tubes into a larger diameter SWCNT, due to the mismatch of their respective honeycomb structures. The extent of reconstruction required can only be accomplished by using long simulation cells as the ones used in the two examples presented in this paper. In summary, we have demonstrated that no restrictions apply for the coalescence of tubes of different chiralities, provided that the simulation cell in the axial direction of the tubes is long enough. Coalescence proceeds following the same patching-and-tearing mechanism introduced to explain coalescence of non chiral tubes. However, it is fair to recognize that the resulting tubes have higher concentrations of defects than in the case of coalescence of non chiral tubes of the same type. Little or no annealing of those defects is observed within the limited time scale of our simulations.

As we have pointed out above, large simulation cells are used to avoid unphysical uniform distributions of vacancies arising from the repetition of the cell within the periodic boundary conditions scheme and to accommodate the tube reconstruction involved in the coalescence of tubes with different chiralities. However, the use of smaller cells would be preferred from the computational (time saving) view point. We have studied the effect of reducing the size of the simulation cell down to 20 unit cells in the axial direction of the tubes, in the case of coalescence of two non-chiral $(10,10)$ tubes. The use of the smaller cell does not change the outcome of the simulated coalescence and the patching-and-tearing mechanism describing the process remains valid. Therefore in the next section, where we simulate bundles of $(10,10)$ tubes, we will use the smaller simulation cell without any loss of generality or physical insight. 


\subsection{Transformation of SWCNT's ropes into MWCNT's}

Let us first consider a bundle of seven $(10,10)$ tubes arranged in a triangular lattice (with a lattice parameter of $17 \AA$ ) with a central tube and six surrounding tubes (see Fig. 7). For investigating the temperature induced SW-to-MW transformation we assume that, similarly to the case of coalescence of two tubes, the vacancies play the leading role in promoting the structural reorganization of the bundles. This assumption will be lately confirmed by the simulations (see below). The thermal treatment of the bundle at elevated temperatures will produce a number of vacancies in the tubes. Therefore we begin the simulation by generating a number (4\%) of vacancies in the intertube regions defined by the six outer tubes (no defects are created in the central tube, see below for a justification). Upon the generation of vacancies, polymerization between neighboring tubes takes place in less than 1 ps (see Fig. $7, t=1 \mathrm{ps}$ ). The bundle is then progressively heated up. Coalescence between neighboring tubes begins to develop following the patching-and-tearing mechanism described above (see Fig. 7, $t=213 \mathrm{ps}$ ). The concerted coalescence of the tubes around the bundle gives rise to the transformation of the bundle of SWCNTs into a MWCNT consisting of three nested tubes (see Fig. $7, t=245 \mathrm{ps}$ ). The central tube may also have a number of defects. Then, depending on the links established between the central tube and its surroundings, three (as the one shown here) or two nested structures may occur.
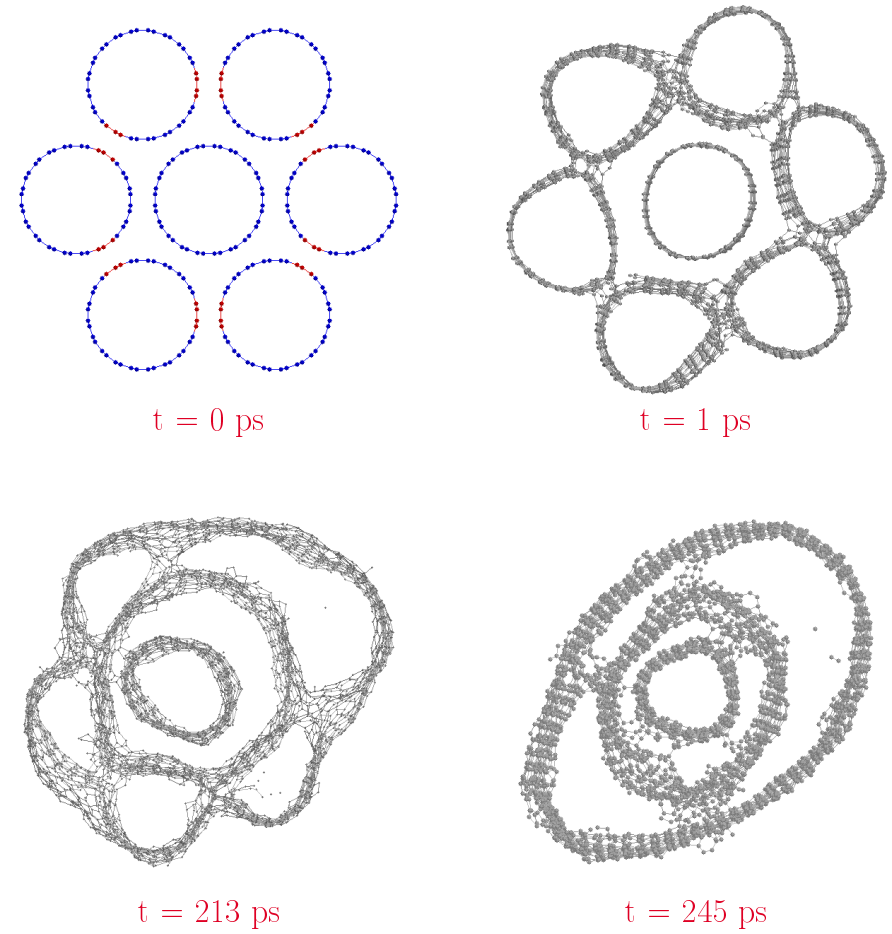

Figure 7. Snapshots (top views) taken from the simulations showing the sequence of the SW-to-MW transformation of a bundle of seven $(10,10)$ tubes.

Bundles containing four and five tubes transform into MWCNTs consisting of two nested tubes (see Fig. 8) following a similar patching-and-tearing mechanism operating in a concerted way around the bundle.

We find that four tubes in the bundle is the lower limit to observe the SW-to-MW transformation. Thus, the concerted coalescence of three $(10,10)$ SWCNTs produces a SWCNT of larger diameter (see Fig. 9). One could argue about the small amount of $\mathrm{C}$ atoms available to reconstruct an internal tube. However, we find that a bundle of three $(20,20)$ tubes (where the supply of $\mathrm{C}$ atoms would be sufficient to reconstruct an internal $(9,9)$ tube) also coalesces in a SWCNT of larger diameter. 

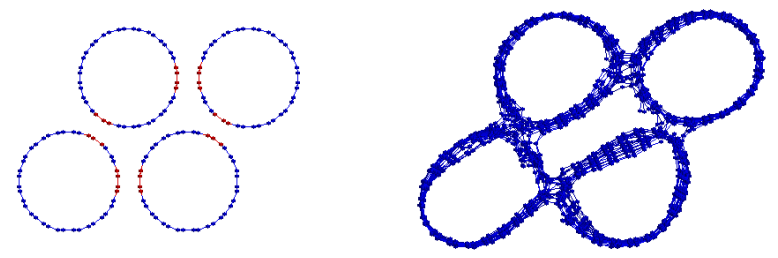

$$
\mathrm{t}=0 \mathrm{ps}
$$

$$
\mathrm{t}=1 \mathrm{ps}
$$

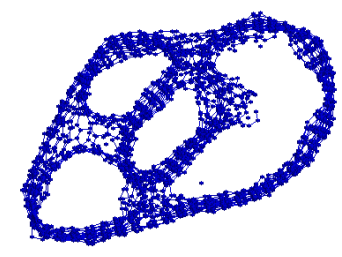

$$
\mathrm{t}=970 \mathrm{ps}
$$

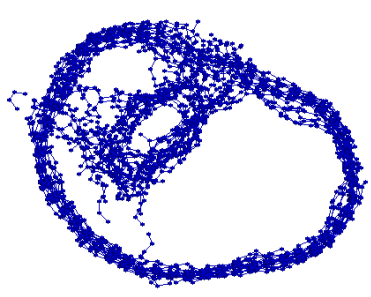

$$
\mathrm{t}=1030 \mathrm{ps}
$$

Figure 8. Snapshots (top views) taken from the simulations showing the transformation of a bundle of four $(10,10)$ tubes into a MWCNT consisting on two nested tubes.

The excellent agreement between the simulations and the experiments gives support for the assumptions made about the role played by the vacancies in the SW-to-MW transformation. We want to stress also that the patching-and-tearing mechanism presented here is of general applicability to describe both the coalescence of SWCNTs into a larger diameter tube and the newest SW-to-MW transformation based on the concerted coalescence of the tubes in a bundle.

\section{SUMMARY}

Novel crystalline ropes of polygonized SWCNTs (tube diameter $D$ about $17 \AA$ ) produced by $\mathrm{CO}_{2}$ laser ablation exhibit rounded-hexagonal cross sections. Simulations of the lattice show several metastable structures characterized by different tube cross sections, hexagonal, rounded-hexagonal and circular, and increasing cell volume. The lowest energy configuration of the lattice progresses from circular to hexagonal with increasing tube diameter. The driving force for the departure of the tubes from the ideal circular shape is the attractive (van der Waals-type) intertube interaction. In contrast to the experimental ropes, the lowest energy configuration of the lattice of tubes with $D=17 \AA$ corresponds to nearly circular tubes. However, the lattice of rounded-hexagonal tubes is very close in energy, what would explain the experimental observation. It would be of great interest to understand and control the production of the different metastable structures of the lattice depending on the growth conditions.

The experimental observation of the transformation of SWCNT bundles into MWCNTs under high temperature treatments motivated us to simulate the thermal stability of the ropes. Extensive Molecular Dynamics simulations combined with the experimental results unveil the physical mechanism of the SW-to-MW transformation based on the patching of SWCNTs and their subsequent tearing apart giving rise to a MWCNT. The driving force for this transformation are the vacancies created by the high temperature treatment. Further studies are required to improve our understanding of the structural stability of the ropes and their possible morphological changes. Possible implications on the different growth regimes of SWCNT bundles are still obscure. 

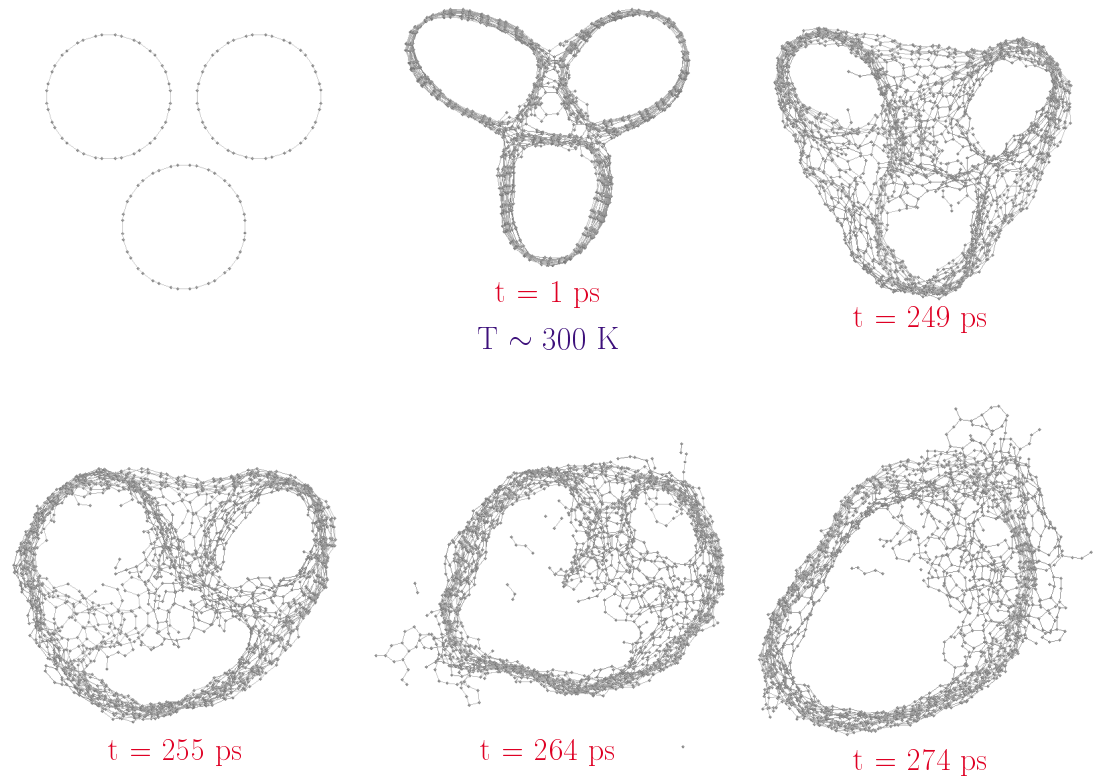

Figure 9. Snapshots (top views) taken from the simulations showing the coalescence of a bundle of three $(10,10)$ tubes into a larger diameter SWCNT.

\section{ACKNOWLEDGMENTS}

The work has been done in collaboration with the groups of S. Iijima (Japon) and Sylvie Bonamy (Orelans, France). Work supported by DGI of Spain (Grant MAT2002-04499-C02), European Community (RTNCOMELCAN), and Junta de Castilla y León (Grant CO01/102). M.J.L. acknowledges support from the Spanish MCYT under the "Ramón y Cajal" Program.

\section{REFERENCES}

1. S. Iijima and T. Ichihashi Nature (London) 363, pp. 603-605, 1993.

2. S. Iijima Nature (London) 354, pp. 56-58, 1991.

3. A. Thess, R. Lee, P. Nikolaev, H. Dai, P. Petit, J. Robert, C. Xu, Y. H. Lee, S. G. Kim, D. Colbert, G. Scuseria, D. Tomanek, J. Fischer, and R. Smalley Science 273, pp. 483-487, 1996.

4. C. Journet, W. K. Maser, P. Bernier, A. Loiseau, M. L. de la Chapelle, S. Lefrant, P. Deniard, R. Lee, and J. E. Fischer Nature (London) 388, pp. 756-758, 1997.

5. T. Hertel, R. E. Walkup, and P. Avouris Phys. Rev. B 58, pp. 13870-13873, 1998.

6. R. S. Ruoff, J. Tersoff, D. C. L. S. Subramoney, and B. Chan Nature 364, pp. 514-516, 1993.

7. J. Tersoff and R. S. Ruoff Phys. Rev. Lett. 73, pp. 676-679, 1994.

8. M. J. López, A. Rubio, J. A. Alonso, L.-C. Qin, and S. Iijima Phys. Rev. Lett. 86, pp. 3056-3059, 2001.

9. M. L. de la Chapelle, S. Lefrant, C. Journet, W. K. Maser, P. Bernier, and A. Loiseau in Molecular Nanostructures, H. Kuzmany, J. Fink, M. Mehring, and S. Roth, eds., p. 387, World Scientific, 1998.

10. K. Méténier, S. Bonnamy, F. Béguin, C. Journet, P. Bernier, M. L. de la Chapelle, O. Chauvet, and S. Lefrant Carbon 40, pp. 1765-1773, 2002.

11. M. J. López, A. Rubio, J. A. Alonso, S. Lefrant, K. Méténier, and S. Bonnamy Phys. Rev. Lett. 89, pp. 255501-1-4, 2002.

12. J. Tersoff Phys. Rev. B 37, pp. 6991-7000, 1988.

13. J. Tersoff Phys. Rev. Lett. 61, pp. 2879-2882, 1988.

14. K. Nordlund, J. Keinonen, and T. Mattila Phys. Rev. Lett. 77, pp. 699-702, 1996. 
15. L.-C. Qin and S. Iijima Chem. Phys. Lett. 269, pp. 65-71, 1997.

16. G. G. Tibbetts J. Cryst. Growth 66, pp. 632-638, 1984.

17. P. Nikolaev, A. Thess, A. G. Rinzler, D. T. Colbert, and R. E. Smalley Chem. Phys. Lett. 266, pp. 422-426, 1997.

18. M. Terrones, H. Terrones, F. Banhart, J.-C. Charlier, and P. M. Ajayan Science 288, pp. 1226-1229, 2000. 